\title{
Transport Properties and a Current-Functional Theory in the Linear-Response Regime
}

\author{
Tai Kai $\mathrm{Ng}$ \\ Department of Physics, Massachusetts Institute of Technology, Cambridge, Massachusetts 02139 \\ (Received 1 September 1988)
}

\begin{abstract}
The possibility of formulating a time-dependent current-functional theory which describes the linear responses of systems of inhomogeneous, interacting electron gas to an arbitrary form of time-dependent electromagnetic field is studied. A Kohn-Sham-type theory for the dynamical electronic conductivity tensor is derived and a local-density type of approximation for the resulting exchange-correlation electric field is constructed.

PACS numbers: $71.10 .+x, 31.20 .5 y$
\end{abstract}

Since the original papers of Hohenberg and Kohn ${ }^{1}$ and Kohn and Sham, ${ }^{2}$ density-functional theory has became one of the most widely used tools in the treatment of electron-electron interaction in condensed matter physics. ${ }^{3,4}$ Recently, the formulation has been generalized to systems under large, static magnetic field in the form of a current-density-functional theory, ${ }^{5}$ and also to the time-dependent linear-response regime under a very general thermodynamic condition. ${ }^{6}$ The content of this Letter is divided into two parts. In the first part, we show that the time-dependent density-functional theory of $\mathrm{Ng}$ and Singwi, ${ }^{6}$ which describes linear responses of electronic systems to external time-dependent longitudinal electric fields, can be generalized to describe (linear) responses of electronic systems to an arbitrary form of time-dependent electromagnetic field, in the form of a current-functional theory. The consequences of this result are discussed. In the second part, we formulate a Kohn-Sham-type theory for the dynamical electronic conductivity tensor and construct a "local-density" type of approximation for the resulting exchange-correlation electric field. Some general comments are also given.

A time-dependent electromagnetic field can be specified by its electric field component, $\mathbf{E}(\mathbf{r}, t)$ $=\mathbf{E}(\mathbf{r}, \omega) e^{i \omega t}$ [notice that $i \omega \mathbf{B}(\mathbf{r}, \omega)=-c \nabla \times \mathbf{E}(\mathbf{r}, \omega)$ from Maxwell's equation, thus the magnetic field is also specified], up to an unimportant gauge factor. We shall prove the following theorem: For a system with fixed number of electrons, there is a one-to-one mapping between the induced current distribution $\mathbf{j}(\mathbf{r}, \omega)$ and the applied electric field $\mathbf{E}(\mathbf{r}, \omega)$ for nonzero $\omega$ in the linearresponse regime if the system under consideration obeys the second law of thermodynamics, and is at stable thermal equilibrium. (The work done by the external potential $\Delta W \geq 0$ to second order of the external potential. $^{7}$ )

Notice that in the linear-response regime, we can generally write

$$
j_{i}(\mathbf{r}, \omega)=\sum_{j} \int \sigma_{i j}^{\prime}\left(\mathbf{r}, \mathbf{r}^{\prime}, \omega\right) E_{j}(\mathbf{r}, \omega) d \mathbf{r}^{\prime},
$$

where $\sigma_{i j}^{\prime}$ is the conductivity tensor for the external elec- tric field and $i, j=x, y, z$ are direction indices. To prove that there is a one-to-one mapping between $\mathbf{j}$ and $E$, it is sufficient to prove that there exists no external field $\mathbf{E}^{\prime}(\mathbf{r}, \omega)$ such that ${ }^{6}$

$$
0 \equiv \sum_{j} \int \sigma_{i j}^{\prime}\left(\mathbf{r}, \mathbf{r}^{\prime}, \omega\right) E_{j}^{\prime}\left(\mathbf{r}^{\prime}, \omega\right) d \mathbf{r}^{\prime} .
$$

To prove this theorem, we follow Ref. 6 and consider the total work done by the external potential, ${ }^{8}$

$$
\begin{array}{r}
\Delta W=\int d t \int d \mathbf{r}\left\{\mathbf{j}(\mathbf{r}, t) \cdot \frac{-1}{c} \frac{\partial \mathbf{A}(\mathbf{r}, t)}{\partial t}\right. \\
\left.+n(\mathbf{r}, t) \frac{\partial \phi(\mathbf{r}, t)}{\partial t}\right\},
\end{array}
$$

where $\mathbf{A}$ and $\phi$ are the external vector and scalar electromagnetic potentials and $\mathrm{j}$ and $n$ are the induced current and density fluctuations. Integrating by parts and using the continuity equation on the second term, we obtain after Fourier transforming in time

$$
\Delta W=\int \frac{d \omega}{2 \pi} \int d \mathbf{r} \mathbf{j}(\mathbf{r}, \omega) \cdot \mathbf{E}(\mathbf{r}, \omega),
$$

where $\mathbf{j}$ is given by Eq. (1) to second order in $\mathrm{E}$.

From thermodynamics, $\Delta W \geq 0$. The equality sign holds only for reversible processes, which are quasistatic (i.e., $\omega=0$ ). For $\omega \neq 0$ processes, $\Delta W>0$ which implies from Eqs. (1) and (4) that $\mathbf{j}(\mathbf{r}, \omega)$ cannot be identically zero in Eq. (1), as long as the external field perturbs the system. This completes the proof of the theorem.

Naively, one expects as a consequence of this theorem that a Kohn-Sham-type theory can be constructed for the (linear) current responses of the system to external time-dependent electromagnetic fields. However, notice that a fundamental assumption we have made in proving the present theorem is that the system under consideration obeys the second law of thermodynamics, whereas the Hohenberg-Kohn-Sham theory involves the Schrödinger equation only. The second law of thermodynamics requires the existence of time-irreversible processes whereas the Schrödinger equation is time reversible. Thus, the present theorem does not necessarily im- 
ply the existence of one-to-one mapping between induced current distribution and applied external electromagnetic field for a system which obeys the Schrödinger equation only (see Refs. 7 and 9 for an example). In particular, it does not guarantee the validity of a straightforward Kohn-Sham-type of approach (it is not certain that the Kohn-Sham approach obeys the second law of thermodynamics).

In practice, this difficulty can be overcome by introducing a small imaginary frequency to the response function evaluated in the Kohn-Sham theory; i.e., $\omega \rightarrow \omega+i \delta$ in $\sigma_{i j}^{\prime}\left(\mathbf{r}, \mathbf{r}^{\prime}, \omega\right)$, where $\delta>0$, which phenomenologically couples the system to an external heat bath, and our theorem is valid as long as $\delta>0$. (In fact, this result can be proved directly from the Kubo formula for the current-current response function, ${ }^{10}$ without involving directly the second law of thermodynamics.) Thus a Kohn-Sham-type theory can be constructed for finite $\delta$, of which the current-current correlation function can be computed, and the physical result can be obtained by analytic continuation (extrapolation) to the $\delta \rightarrow 0$ limit. Notice that this procedure is actually the standard one of which a retarded response function is being defined. Thus our analytic continuation procedure gives the correct result as long as the standard procedure of defining a retarded response function remains valid.

With this procedure in mind, we now turn to the formulation of the one-particle Kohn-Sham equation. First, we make the necessary assumption that the density distribution function $n_{0}(\mathbf{r})+n(\mathbf{r}, \omega)$ and current distribution function $\mathbf{j}_{0}(\mathbf{r})+\mathbf{j}(\mathbf{r}, \omega)$, where $n_{0}(\mathbf{r})$ and $\mathbf{j}_{0}(\mathbf{r})$ are the density and current distribution functions of the system in the absence of the time-dependent perturbing potential [here we shall only consider nonmagnetic systems such that $\left.\mathbf{j}_{0}(\mathbf{r}) \equiv 0\right],{ }^{5}$ are $v$ representable; ${ }^{11}$ i.e., they can be reproduced by a system of noninteracting electrons moving in appropriate single-particle potentials $\phi_{\mathrm{eff}}(\mathbf{r})$ and electric field $\mathbf{E}_{\mathrm{eff}}(\mathbf{r}, \omega)$. Notice that the separation of the current and density variables into a dominant $\omega=0$ part plus small time-dependent fluctuations is a special feature of the linear-response regime only, and is not possible in a general time-dependent theory. Notice also that in the limit $\mathbf{E}(\mathbf{r}, \omega)=0, \phi_{\mathrm{eff}}(\mathbf{r})$ must reproduce the correct ground-state density distribution $n_{0}(r)$ and is thus the ordinary Kohn-Sham potential. For $\omega \neq 0$, $n(\mathbf{r}, \omega)$ is completely determined by $\mathbf{j}(\mathbf{r}, \omega)$ by the continuity equation. Therefore it is sufficient to consider only the induced current $\mathbf{j}(\mathbf{r}, \omega)$. From the linearresponse requirement, we must have

$$
j_{i}(\mathbf{r}, \omega)=\sum_{j} \int d \mathbf{r}^{\prime} \sigma_{i j}^{\mathrm{KS}}\left(\mathbf{r}, \mathbf{r}^{\prime}, \omega\right) E_{\mathrm{eff}_{j}}\left(\mathbf{r}^{\prime}, \omega\right)
$$

where $\sigma^{\mathrm{KS}}$ is the conductivity tensor for a system of noninteracting electrons moving in the Kohn-Sham potential $\phi_{\text {eff }}(\mathbf{r})$. The effective electric field $\mathbf{E}_{\text {eff }}$ can be written as

$\mathbf{E}_{\mathrm{eff}}(\mathbf{r}, \omega)=\mathbf{E}(\mathbf{r}, \omega)+\nabla_{\mathbf{r}} \int d \mathbf{r}^{\prime} \frac{n\left(\mathbf{r}^{\prime}, \omega\right)}{\left|\mathbf{r}-\mathbf{r}^{\prime}\right|}+\mathbf{E}_{\mathrm{xc}}(\mathbf{r}, \omega)$,

where $\mathbf{E}(\mathbf{r}, \omega)$ is the external electric field and the second term is the polarization field. The first and second terms on the right-hand side of Eq. (6) together form the total electric field $\mathbf{E}_{t}(\mathbf{r}, \omega)$ felt by electrons in the system. The exchange-correlation electric field $\mathbf{E}_{\mathrm{xc}}(\mathbf{r}, \omega)$ is defined by Eqs. (5) and (6) and incorporates all other interaction effects. The exact form of $\mathbf{E}_{\mathrm{xc}}$ is unknown except that from the existence theorem, we know that it can be expressed as linear functional of $\mathbf{j}(\mathbf{r}, \omega)$,

$$
E_{\mathrm{xc} i}(\mathbf{r}, \omega)=\sum_{k} \int d \mathbf{r}^{\prime} \xi_{i k}\left(\mathbf{r}, \mathbf{r}^{\prime}, \omega\right) j_{k}(\mathbf{r}, \omega),
$$

where $\xi_{i k}$ is a functional of the unperturbed ground-state particle density $n_{0}(\mathbf{r})$. Thus Eqs. (5)-(7) together with the continuity equation define a scheme where the induced current fluctuation $\mathbf{j}(\mathbf{r}, \omega)$ and density fluctuation $n(\mathbf{r}, \omega)$ can be determined self-consistently. Notice that in general, we do not know how to construct the tensor $\xi_{i k}$ except that it can be formally defined through Eqs. (1) and (5)-(7) as

$$
\xi\left(\mathbf{r}, \mathbf{r}^{\prime}, \omega\right)=\sigma^{\mathrm{KS}}\left(\mathbf{r}, \mathbf{r}^{\prime}, \omega\right)^{-1}-\boldsymbol{\sigma}\left(\mathbf{r}, \mathbf{r}^{\prime}, \omega\right)^{-1},
$$

where $\sigma\left(\mathbf{r}, \mathbf{r}^{\prime}, \omega\right)$ is the (exact) usual conductivity tensor for the interacting system which relates the induced current distribution to the total electric fields; i.e., $\mathbf{j}=\boldsymbol{\sigma} \cdot \mathbf{E}_{t}$ and $\boldsymbol{\sigma}^{\mathrm{KS}}$ is the corresponding quantity for the system of noninteracting electrons moving in the KohnSham potential, provided that these inverse functions exist.

We now proceed to the discussion of the local-density type of approximation for $\xi$. For this purpose we first consider $\xi$ for the homogeneous $\left[n_{0}(\mathbf{r})=\right.$ const] situation.

For homogeneous systems, the conductivity tensor $\sigma$ has the general form

$\sigma_{i j}^{h}(\mathbf{q}, \omega)=\sigma_{T}(q, \omega)\left[\delta_{i j}-\frac{q_{i} q_{j}}{q^{2}}\right]+\sigma_{L}(q, \omega) \frac{q_{i} q_{j}}{q^{2}}$,

where $\sigma_{T}$ and $\sigma_{L}$ are scalar functions describing the response of the system to transverse and longitudinal electric fields, respectively. Thus from Eq. (8), we obtain for homogeneous systems

$$
\begin{aligned}
\xi_{i j}^{h}(\mathbf{q}, \omega)= & {\left[\frac{1}{\sigma_{T}^{0}(q, \omega)}-\frac{1}{\sigma_{T}(q, \omega)}\right]\left(\delta_{i j}-\frac{q_{i} q_{j}}{q^{2}}\right) } \\
& +\left[\frac{1}{\sigma_{L}^{0}(q, \omega)}-\frac{1}{\sigma_{L}(q, \omega)}\right] \frac{q_{i} q_{j}}{q^{2}} \\
= & \xi_{T}(q, \omega)\left(\delta_{i j}-\frac{q_{i} q_{j}}{q^{2}}\right)+\xi_{L}(q, \omega) \frac{q_{i} q_{j}}{q^{2}},
\end{aligned}
$$

where $\sigma_{L(T)}^{0}$ is the conductivity tensor for the corresponding noninteracting electron gas. The longitudinal con- 
ductivity $\sigma_{L}(q, \omega)$ is related to the scalar dielectric function $\epsilon(q, \omega)$ through

$$
\begin{aligned}
\epsilon(q, \omega) & =1+\frac{4 \pi i \sigma_{L}(q, \omega)}{\omega} \\
& =1-\frac{v_{q} \chi_{0}(q, \omega)}{1+v_{q} G(q, \omega) \chi_{0}(q, \omega)},
\end{aligned}
$$

where $\chi_{0}(q, \omega)$ is the Lindhard function and $G(q, \omega)$ is the so-called local-field factor. ${ }^{11,12}$ From Eqs. (10) and (11), we obtain

$$
\xi_{L}(q, \omega)=\frac{q^{2}}{i \omega} v_{q} G(q, \omega) .
$$

The $q \rightarrow 0, \omega \gg q$ limit of $\xi_{L(T)}(q, \omega)$ is of particular interest since it will enter in the local-density type of approximation. In the longitudinal case, it is known that $v_{q} G(q, \omega) \rightarrow \alpha_{L}(\omega)$ as $q \rightarrow 0,{ }^{11-13}$ where $\alpha_{L}(\omega)$ is finite as $\omega \rightarrow 0$, so that $\xi_{L}(q, \omega) \rightarrow q^{2} \alpha_{L}(0) / i \omega$ in this limit. The transverse conductivity is also known to have the form ${ }^{14,15}$ (see also Ref. 5)

$$
\sigma_{T}(q, \omega) \sim \frac{i n e^{2}}{m \omega}\left(1+q^{2} \frac{\alpha_{T}(\omega)}{\omega^{2}}\right),
$$

$$
\begin{aligned}
I_{\mathrm{xc}}^{h} & =\frac{1}{2} \int d \mathbf{q}\left\{\xi_{L}(q, \omega)\left|\mathbf{j}_{L}(q, \omega)\right|^{2}+\xi_{T}(q, \omega)\left|\mathbf{j}_{T}(q, \omega)\right|^{2}\right\} \\
& =\frac{1}{2} \int d \mathbf{q}\left\{\frac{\xi_{L}(q, \omega)}{q^{2}}[\mathbf{q} \cdot \mathbf{j}(q, \omega)]^{2}+\frac{\xi_{T}(q, \omega)}{q^{2}}[\mathbf{q} \times \mathbf{j}(q, \omega)]^{2}\right\},
\end{aligned}
$$

where $\mathbf{j}_{L}$ and $\mathbf{j}_{T}$ are the longitudinal and transverse parts of the current, respectively. Notice that $\xi_{L} / q^{2}$ and $\xi_{T} / q^{2}$ are nonsingular functions in the $q \rightarrow 0(\omega \neq 0)$ limit.

Equation (16) suggests that one can make the following local-density type of approximation for $I_{\mathrm{xc}}$ in the general, inhomogeneous situation (see also Ref. 11). We assume that (i) the local density $n_{0}(\mathbf{r})$ is a slowly varying function in space so that one can replace the function $\xi$ by the corresponding function $\xi^{h}$ for a homogeneous electron gas locally, and (ii) the induced current distribution $\mathrm{j}(\mathbf{r}, \omega)$ also varies slowly in space so that only the $q \rightarrow 0$ limit of $\xi^{h}$ needs to be considered.

With these assumptions, we arrive at the following approximation:

$$
I_{\mathrm{xc}} \sim \frac{1}{2} \int d r\left\{\xi_{L}^{*}\left(\omega ; n_{0}(\mathbf{r})\right)|\nabla \cdot \mathbf{j}(\mathbf{r}, \omega)|^{2}+\xi_{T}^{*}\left(\omega ; n_{0}(\mathbf{r})\right)|\nabla \times \mathbf{j}(\mathbf{r}, \omega)|^{2}\right\},
$$

where

$$
\xi_{L(T)}^{*}(\omega ; n)=\lim _{q \rightarrow 0} \frac{\xi_{L(T)}(q, \omega)}{q^{2}},
$$

for a homogeneous electron gas with density $n$. The exchange-correlation electric field is given from straightforward functional derivatives of $I_{\mathrm{xc}}$ [Eq. (15)] by

$$
\mathbf{E}_{\mathrm{xc}}(\mathbf{r}, \omega)=-\nabla\left\{\xi_{L}^{*}\left(\omega ; n_{0}(\mathbf{r})\right) \nabla \cdot \mathbf{j}(\mathbf{r}, \omega)\right\}-\nabla \times\left\{\xi_{T}^{*}\left(\omega ; n_{0}(\mathbf{r})\right) \nabla \times \mathbf{j}(\mathbf{r}, \omega)\right\} .
$$

Equations (5), (6), and (18) now form a self-consistent, approximate scheme in which the dynamical conductivity of electronic systems can be calculated. Notice that in the case $\nabla \times j \equiv 0$ (i.e., only longitudinal current present), we have [using Eq. (12) and the continuity equation]

$$
\mathbf{E}_{\mathrm{xc}}(\mathbf{r}, \omega)=-\nabla\left\{\lim _{q \rightarrow 0}\left[v_{q} G\left(q, \omega ; n_{0}(\mathbf{r})\right)\right] n(\mathbf{r}, \omega)\right\}
$$

in agreement with the approximate expression for the exchange-correlation potential derived by Gross and Kohn ${ }^{11}$ for the case of a time-dependent scalar perturbing potential. An approximate expression for $\xi_{L}^{*}$ [or $\left.v_{q} G(q, \omega)\right]$ has been ob- 
tained by these authors, which is convenient to use. The corresponding approximation for $\xi_{T}^{*}$ is still missing and is at present under study.

We would now like to make a few comments about our formulation, and about the local-density approximation.

It is important to keep in mind that our formulation is valid only for frequencies $\omega \neq 0$, where $\Delta W>0$. In fact, Vignale and Rasolt ${ }^{5}$ have shown that for electrons in static, external potentials, the ground state is a functional of the static density $n_{0}(r)$ and paramagnetic current $\mathbf{j}_{p}(\mathbf{r})$, instead of just the total current $\mathbf{j}(\mathbf{r})$. This difference arises because in the static case, the $\mathbf{E}$ and $\mathbf{B}$ fields are independent, and so are the particle density $n_{0}(\mathbf{r})$ and current density $\mathbf{j}(\mathbf{r})$. However, in the dynamic case, the $\mathbf{B}$ field is completely determined by the $\mathbf{E}$ field via the Maxwell equation, and the density fluctuation $n(\mathbf{r}, \omega)$ is completely determined by $\mathrm{j}(\mathbf{r}, \omega)$ via the continuity equation. Thus the nonequilibrium fluctuation is completely determined by the $\mathbf{E}$ field, or current $\mathbf{j}$, in contrast to the ground-state situation.

We would also like to make a few comments on our local-density approximation. An implicit assumption we have made in defining $\xi_{L(T)}^{*}(\omega ; n)$ [Eq. (17)] is that $q \ll \omega$ in the functions $\xi_{L(T)}(q, \omega)$. However, the existence of nonzero $\nabla \cdot \mathbf{j}(\mathbf{r})$ or $\nabla \times \mathbf{j}(\mathbf{r}, \omega)$ terms implies that there exists a characteristic nonzero wave vector $q_{0}(r)$ which measures the "size" of the divergence or curl of the current at any local space point $\mathbf{r}$ and our approximation for $\xi_{L(T)}$ is valid only when $\omega \gg q_{0}(\mathbf{r})$. This is not expected to be always satisfied for a realistic system under low-frequency external perturbation and therefore our approximation is expected to be satisfactory only for the calculation of high-frequency conductivity. In particular, our local-density approximation for $\xi_{L(T)}$ is not expected to be satisfactory for the calculation of dc conductivities. The approximate method for calculating dc conductivities has been constructed in the case of electrons moving in weakly disordered potentials and will be discussed elsewhere.

In summary, we have shown that under a very general thermodynamic condition (or in practice, letting $\omega \rightarrow \omega+i \delta$, with $\delta>0$ in the response function), a current-functional theory can be constructed to compute transport properties of electronic systems in the linearresponse regime. A local-density type of approximation for the resulting exchange-correlation electric field is constructed for this purpose. We have also pointed out that there are strong limitations to our local-density type of approximation, which demands care in realistic applications.

The author thanks G. Vignale for helpful discussions. This work is supported by the National Science Foundation under Grant No. DMR-8521377.

${ }^{1}$ P. Hohenberg and W. Kohn, Phys. Rev. 136, B864 (1964).

${ }^{2}$ W. Kohn and L. J. Sham, Phys. Rev. 140, A1133 (1965).

${ }^{3}$ For a review, see Theory of Inhomogeneous Electron Gas, edited by S. Lundqvist and N. H. March (Plenum, New York, 1983).

${ }^{4}$ See, for example, Density Functional Methods in Physics, edited by R. M. Dreizler and J. da Providencia, NATO Advanced Study Institute, Ser. B, Vol. 123 (Plenum, New York, 1985).

${ }^{5}$ G. Vignale and M. Rasolt, Phys. Rev. Lett. 59, 2360 (1987).

${ }^{6}$ T. N. Ng and K. S. Singwi, Phys. Rev. Lett. 59, 27 (1987).

${ }^{7}$ E. K. U. Gross, D. Mearns, and L. N. Olivera, Phys. Rev. Lett. 61, 1518 (1988); T. K. Ng and K. S. Singwi, Phys. Rev. Lett. 61, 1519 (1988).

${ }^{8}$ See, for example, D. Forster, Hydrodynamic Fluctuations, Broken Symmetry, and Correlation Functions (Benjamin, New York, 1983).

${ }^{9}$ D. Mearns and W. Kohn, Phys. Rev. A 35, 4796 (1987).

${ }^{10} \mathrm{~T} . \mathrm{K} . \mathrm{Ng}$ (unpublished); the proof proceeds in a way very similar to the proof of the invertibility of the zero-frequency density-density response function by Mearns and Kohn (Ref. 9).

${ }^{1}$ E. K. U. Gross and W. Kohn, Phys. Rev. Lett. 55, 2850 (1985).

${ }^{12}$ K. S. Singwi and M. P. Tosi, in Solid State Physics, edited by H. Ehrenreich, F. Seitz, and D. Turnbull (Academic, New York, 1981), Vol. 36.

${ }^{13}$ See, for example, S. Ichimaru, Rev. Mod. Phys. 54, 1017 (1982).

${ }^{14}$ D. Pines and P. Nozieres, The Theory of Quantum Liquids (Benjamin, New York, 1966).

${ }^{15} \mathrm{P}$. Nozieres, The Theory of Interacting Fermi Systems (Benjamin, New York, 1964).

${ }^{16}$ E. Runge and E. K. U. Gross, Phys. Rev. Lett. 52, 997 (1984).

${ }^{17}$ V. Peuckert, J. Phys. C 11, 4945 (1978). 